\title{
Author's reply to Wong and De Murtinho-Braga
}

\author{
Jacques D Donzé research associate \\ Division of General Internal Medicine, Bern University Hospital, 3010 Bern, Switzerland
}

Wong was worried about the misinterpretation of odds and risks. ${ }^{12}$ As everyone knows, the interpretation of risk is more intuitive than for odds, for both doctors and patients. Although statistically odds are not risks, it is well accepted that odds ratio is a good approximation of the relative risk when the outcome is rare $(<10 \%) .{ }^{3}$ In our study, the outcome was rare $(1.5 \%)$, and therefore relative risk reflected the odds ratio well. A twofold increase in the odds can therefore be expressed as a twofold increase in risk. We also took care to mention the exact odds ratio when the probability was presented as a risk.

In response to De Murtinho-Braga, ${ }^{4}$ number needed to treat (NNT) or number needed to harm (NNH) are appealing summary statistics because they are easy to understand. Nonetheless, they also have major undesirable properties. ${ }^{5}$ Firstly, there is no finite value, and no single value of NNT or NNH that corresponds to no difference. Secondly, these measures do not tell the reader whether there is a statistically significant difference or not.

Thirdly, NNT can be easily calculated in randomised control trials in which by definition no adjustment for confounding factors is necessary since the exposed and non-exposed groups are similar. In cohort studies, however, a direct comparison of proportion (as absolute risk reduction and $\mathrm{NNH}$ ) would give biased results when not adjusted for confounders. Furthermore, the $\mathrm{NNH}$ of 230 is misleading, since it is limited to the pulmonary embolism outcome only. The NNH for all postoperative thrombotic events in our study is 34 . It is also worth noting that this NNH would decrease rapidly as the severity of the sepsis increases.

In conclusion, the best summary statistic to be presented in a cohort study is still a matter of debate.

\section{Competing interests: None declared}

1 Wong D. Conflating odds ratio with "risk." BMJ 2014:349:g6291

2 Donzé JD, Ridker PM, Finlayson SRG, Bates DW. Impact of sepsis on risk of postoperative arterial and venous thromboses: large prospective cohort study. BMJ 2014;349:g5334. (8 September.)

3 De Murtinho-Braga J. Using clinically relevant measures of risk. BMJ 2014;349:g6293.

4 Sedgwick P. Relative risks versus odds ratios. BMJ 2014;348g1407.

5 Hutton JL. Number needed to treat and number needed to harm are not the best way to report and assess the results of randomised clinical trials. Br J Haematol 2009; 146:27-30. 